\title{
A Nanorobotics - The Changing Face of Dentistry
}

\author{
Dr. Mehra $\mathbf{P}^{1}$, Dr. Nabhi $\mathbf{K}^{2}$ \\ ${ }^{1}$ Post Graduate Student, Department of Periodontology, Bhojia Dental College and Hospital, Baddi \\ ${ }^{2}$ Post Graduate Student, Department of Endodontics, Bhojia Dental College and Hospital, Baddi
}

\begin{abstract}
Nanotechnology is the manipulation of matter on the molecular and atomic levels. It has the potential to bring enormous changes into the fields of medicine and dentistry. A day may soon come when nanodentistry will succeed in maintaining near-perfect oral health through the aid of nanorobotics, nanomaterials and biotechnology. However, as with all developments, it may also pose a risk for misuse. Time, economical and technical resources, and human needs will determine the direction this revolutionizing development may take. This article reviews the current status and the potential clinical applications of nanotechnology, nanaomedicine and nanodentistry.
\end{abstract}

Keywords: Nanodentistry, nanomaterials, nanorobots, nanotechnology

\section{Introduction}

The word nano originates from the Greek word "dwarf". The concept of nanotechnology was first elaborated in 1959 by Richard Feynman, a Nobel Prize winning physicist, in a lecture titled, "There's plenty of room at the bottom". He ended the lecture concluding "this is a development which I think cannot be avoided ${ }^{1 \text { ", }}$. Since then, nanotechnology has found use in a myriad of applications including dental diagnosis, material and therapeutics. A day may soon come when nanodentistry will succeed in maintaining near-perfect oral health through the aid of nanorobotics, nanomaterials and biotechnology $y^{2}$. Nanotechnology is the science of manipulating matter measured in the billionths of meters or nanometer, roughly the size of 2 or 3 atoms. The growing interest in the future of dental applications of nanotechnology lead to the emergence of nanodentistry which involves the maintenance of oral health by the use of nanomaterials,biotechnology and dental nanorobotics. According to nanorobotic theory, "nanorobots are microscopic in size, it would probably be necessary for very large numbers of them to work together to perform microscopic and macroscopic tasks"3.

\section{Nanotechnology has applications in many fields like ${ }^{4}$ :}
a) Medicine
1) Diagnostics
2) Drug delivery
3) Tissue engineering

b) Chemistry and environment

1) Catalysis

2) Filtration

c) Energy

1) Reduction of energy consumption
2) Increasing the efficiency of energy production

3) Recycling of batteries

d) Information and communication

1) Novel semiconductor devices

2) Novel optoelectronic devices

3) Displays

4) Quantum Computers

e) Heavy Industry

1) Aerospace

2) Refineries

3) Vehicle manufacturers

4) Consumer goods

5) Foods.

History

One of the first mentions of the distinguishing concepts in nanotechnology (but predating the use of that name) was in 1867. At that time, James Clerk Maxwell proposed, as a thought experiment, a tiny entity known as Maxwell's Demon that was able to handle individual molecules. The term nanotechnology was coined by Prof. Kerie E. Drexler, a researcher and writer of nanotechnology. Humans have been using nanotechnology for a long time without realizing it. The processes of making steel, vulcanizing rubber and sharpening a dental instrument all rely on manipulations of nanoparticles. Richard Zsigmondy studied nanomaterials in the early 20th century, and later discoveries culminated in ideas presented by Nobel Prize winning physicist Richard Feynman in a lecture called "Plenty of Room at the Bottom" in 1959, in which he explored the implications of matter manipulation. 8 Applications began in the 1980s with the invention of the scanning tunneling microscope and the discovery of carbon nanotubes and fullerenes ${ }^{3}$. 


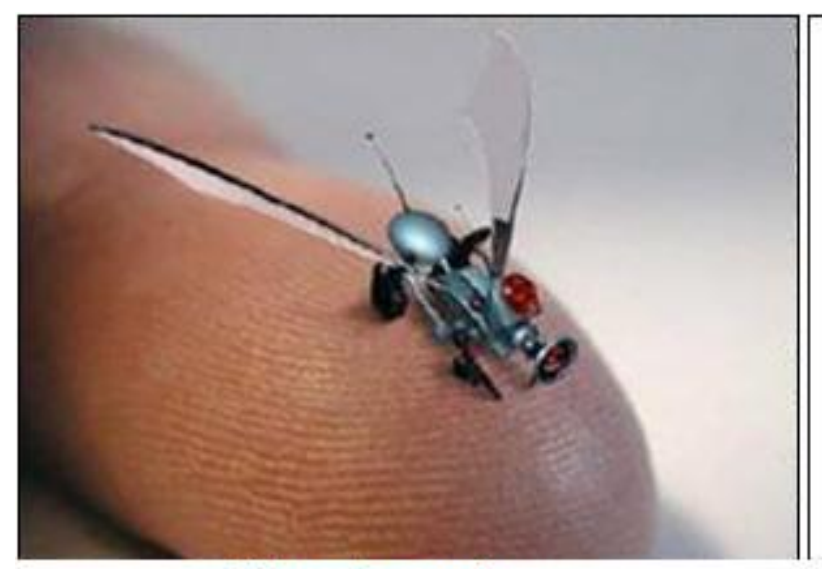

Figure 1: nanorobot

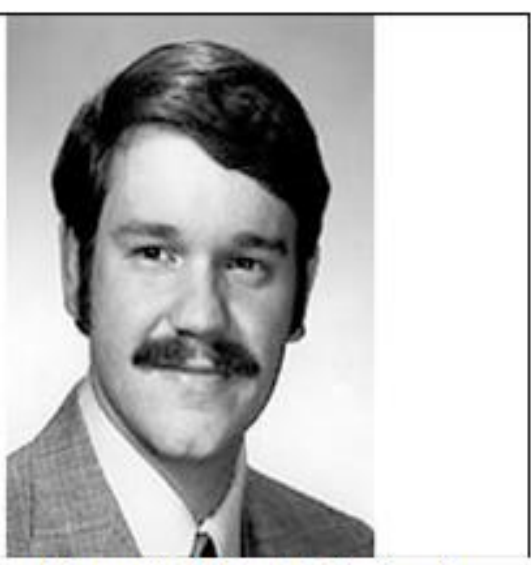

Figure 2: Robert A. Freitas Jr

\section{Nanorobots}

A nanorobot is essentially a controllable machine at the nanometer (A nanometer is a billionth part of a meter, i.e., about $1 / 80,000$ of the diameter of a human hair. Scale of nanometer ranges from $1-100 \mathrm{~nm}$ ) or molecular scale that is composed of nano-scale components and algorithmically responds to input forces and information ${ }^{5}$.
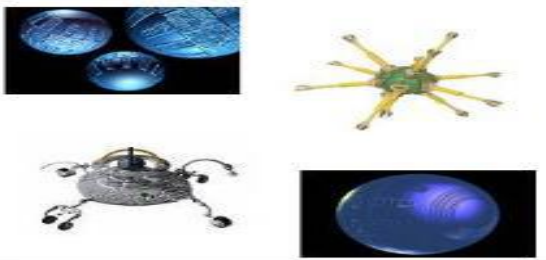

Figure 3: Nanorobots

Nanorobots would constitute any active structure (nanoscale) capable of actuation, sensing, signalling, information processing, intelligence, and swarm behaviour at nano-scale. The nanorobots are invisible to the naked eye, techniques like scanning electron microscopy (SEM) and atomic force microscopy (AFM) are being employed to visualize these nano-scaled devices. These nanorobots provide benefits in numerous areas such as synthesis of new materials with advanced properties, production technology, information technology, medical applications, transportation, etc ${ }^{5}$.

Because of their advanced properties nanorobots are widely used in the field of medicine where they perform inspections, operations, treatment of diseases in the human body, imaging and delivery of drugs to target cells like cancer cells. The advances of nanorobots in dentistry have been relatively slow in comparison to the medical field. Hence the aim of present review article is to enlighten the applications of nanorobots in the management of dental health. Nanorobotics is a field which calls for collaborative efforts between physicists, chemists, biologists, computer scientists, engineers, and other specialists to work towards this common objective and leads emergence of newer branches like nanomedicine and nanodentistry in future ${ }^{5}$.

\section{Components of Nanorobots ${ }^{3}$}

1) $100 \mathrm{~nm}$ manipulating arms

2) $2.10 \mathrm{~nm}$ sorting rotors for molecular-by-molecular purification

3) Smooth super hard surfaces made of atomically flawless diamond

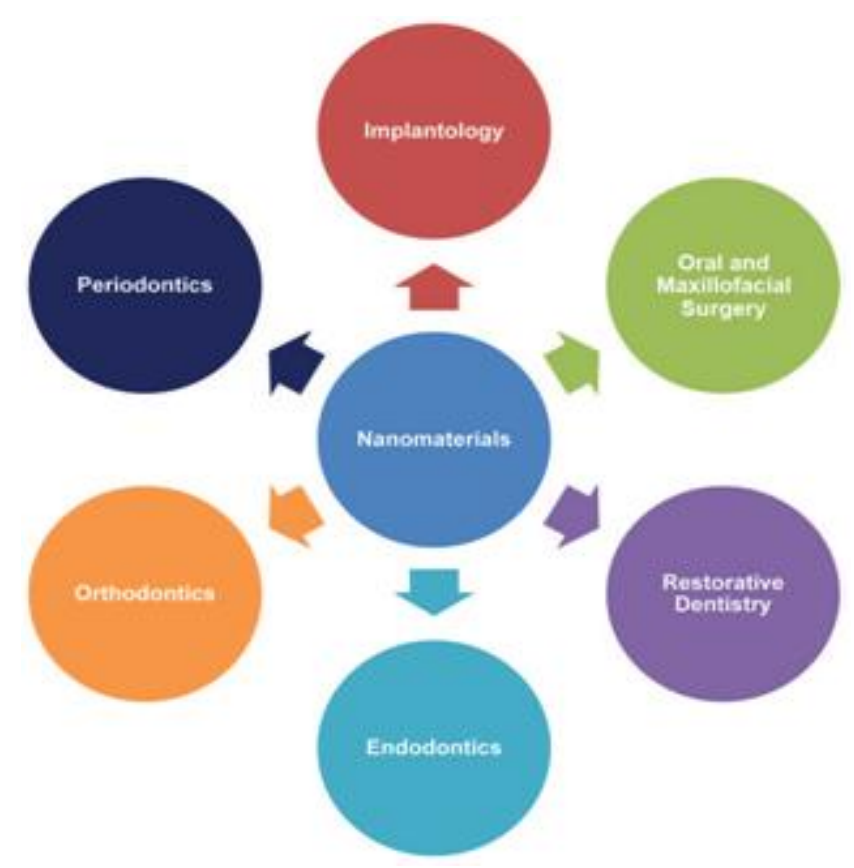

Figure 10: Diagram showing a variety of applications of nanotechnology ${ }^{6}$. Applications of nanotechnology in diagnosis and
treatment $^{7}$

\section{Nanodiagnostics}

Nanodiagnostic devices can be used for early disease identification at the cellular and molecular levels. Nanomedicine could increase the efficiency and reliability of in vitro diagnostics, through the use of selective nanodevices to collect human fluids or tissue samples and to make multiple analyses at the subcellular level. From an in vivo perspective, nanodevices might be inserted into the body to identify the early presence of a disease, or to identify and quantify toxic molecules, tumor cells, and so forth (Freitas, 2000 ; Lampton, 1995 ${ }^{8}$ ). 


\section{International Journal of Science and Research (IJSR) \\ ISSN (Online): 2319-7064 \\ Index Copernicus Value (2013): 6.14 | Impact Factor (2014): 5.611}

\section{Diagnosis and treatment of oral cancer}

Saliva is used as an inexpensive and noninvasively obtained diagnostic medium that contains proteomic and genomic markers for molecular disease identification. Exosome, a membrane-bound secretory vesicle, is one such marker whose level is elevated in malignancy. This marker has been studied by using atomic force microscopy, which employs nanoparticles. The nanoelectromechanical system, oral fluid nanosensor test, and optical nanobiosensor can also be used for diagnosing oral cancer ${ }^{7}$.

Nanoshells, which are miniscule beads, are specific tools in cancer therapeutics. Nanoshells have an outer metallic layer that selectively destroys cancer cells, while leaving normal cells intact. Brachytherapy is an advanced form of cancer treatment. Still under trial are nanoparticle-coated, radioactive sources placed close to or within the tumor to destroy it. Other uses of nanovectors include drug delivery across the blood-brain barrier in the treatment of Alzheimer's and Parkinson's diseases (Song et al., 2004 ${ }^{9}$; Wong, $\left.2006^{10}\right)^{7}$

\section{Nanoanesthesia}

When nanotechnology or nanorobots are used to induce anesthesia, the gingiva of the patient is instilled with a colloidal suspension containing millions of active, analgesic, micron-sized dental robots that respond to input supplied by the dentist. Nanorobots in contact with the surface of the crown or mucosa can reach the pulp via the gingival sulcus, lamina propria, or dentinal tubules. Once in the pulp, they shut down all sensations by establishing control over nerveimpulse traffic in any tooth that requires treatment. After completion of treatment, they restore this sensation, thereby providing the patient with anxiety-free and needleless comfort. The anesthesia is fast-acting and reversible, with no side effects or complications associated with its use (Freitas, 2000) ${ }^{11}$.

\section{Nanosolutions}

Because they produce unique and dispersible nanoparticles, nanosolutions can be used as bonding agents. Homogeneity is ensured, because the adhesive is mixed perfectly every time. Nanoparticles have also been used as sterilizing solutions in the form of nanosized emulsified oil droplets that bombard pathogens (Nagpal et al., 2011) ${ }^{12}$.

\section{Impression materials}

Nanofillers are integrated into vinylpolysiloxanes, producing a unique siloxane impression material that has a better flow, improved hydrophilic properties, and enhanced precision detail (Kumar and Vijayalakshmi, 2006) ${ }^{13}$.

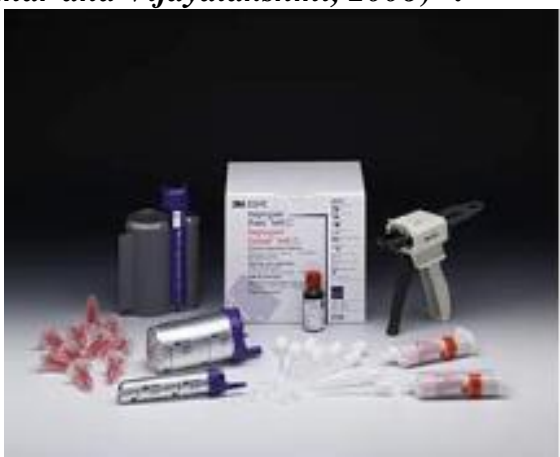

Figure 4: Nano impression material

\section{Bone replacement materials}

Bone is a natural nanostructure that is composed of organic compounds (mainly collagen) and reinforced with inorganic ones. Nanotechnology aims to emulate this natural structure for orthopedic and dental applications and, more particularly, for the development of nanobone. Nanocrystals show a loose microstructure, with nanopores situated between the crystals. The surfaces of the pores are modified such that they adsorb protein, due to the addition of silica molecules. Bone defects can be treated by using these hydroxyapatite nanoparticles (Kumar and Vijayalakshmi, 2006) $)^{13}$.

\section{Hydroxy apatite nanoparticles used to treat bone defects} are $^{14}$ :

- Ostim HA (Osartis GmbH, Germany)

- Vitosso (Orthovita, Inc) HA + TCP (tricalcium phosphate)

- NanOSSTM HA (Angstrom Medica)

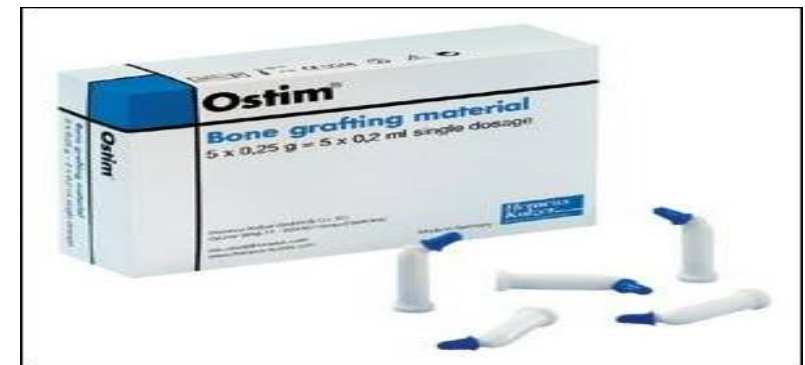

Figure 5: Bone Defects Can Now Be Treated With Hydroxyapetite Nanoparticles

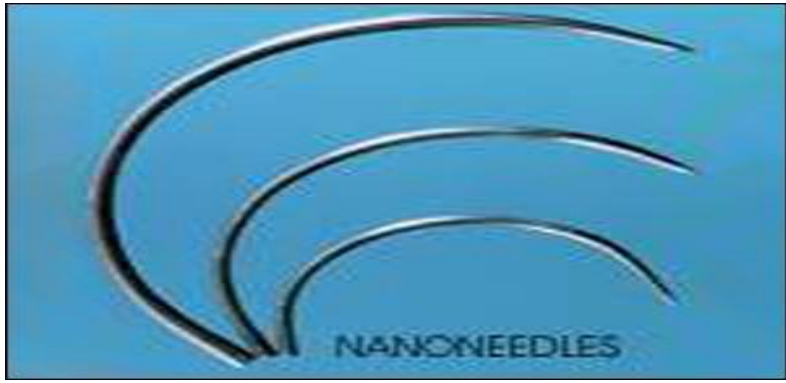

Figure 6: Suture needles with Nano sized stainless steel crystals

\section{Nanoencapsulation}

Targeted release systems that encompass nanocapsules are under trial for inclusion in vaccines and antibiotics (Kumar and Vijayalakshmi, 2006) ${ }^{13}$.

\section{Dentine tubule blocking to alleviate hypersensitivity} Hypersensitivity is caused by changes in the pressure transmitted hydrodynamically to the pulp. The dentinal tubules of a hypertensive tooth have twice the diameter and eight times the surface density of those in nonsensitive teeth. These characteristics have led to the use of nanorobots that selectively and precisely occlude tubules in minutes, by using local, native materials, thus offering patients a quick and permanent cure (Nagpal et al., 2011) ${ }^{12}$.

\section{Nanorobotic dentifrices (Dentifrobots)}

Nanorobotic dentifrices, when delivered either by mouthwash or tooth paste, can cover all subgingival 


\section{International Journal of Science and Research (IJSR) \\ ISSN (Online): 2319-7064 \\ Index Copernicus Value (2013): 6.14 | Impact Factor (2014): 5.611}

surfaces, thereby metabolizing trapped organic matter into harmless and odorless vapors. Properly configured dentifrobots can identify and destroy pathogenic bacteria that exist in the plaque and elsewhere. These invisibly small dentifrobots are purely mechanical devices that safely deactivate themselves when swallowed ${ }^{11}$.

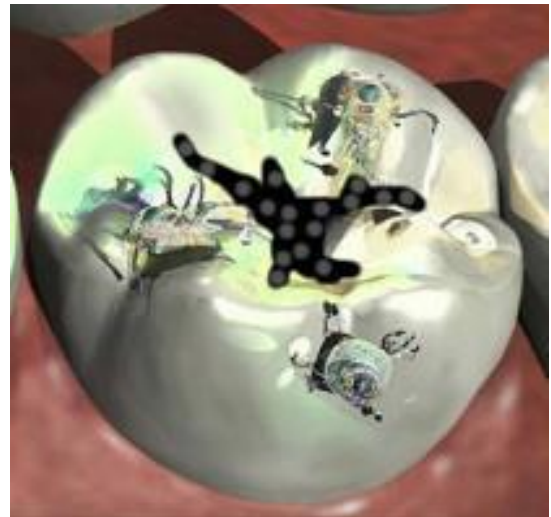

Figure 7: Nanorobots in tooth repair

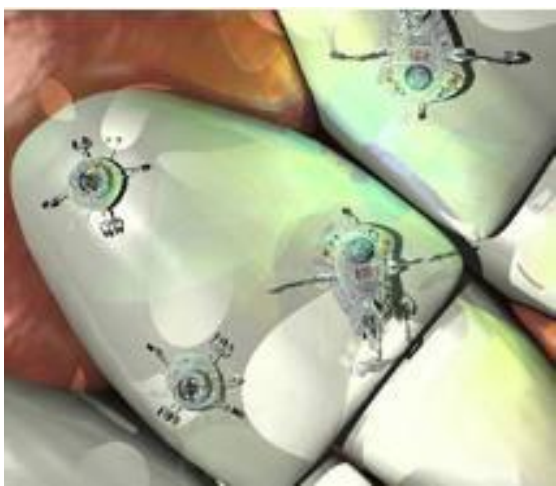

Figure 8: Nanodentifrice

\section{Orthodontics}

Orthodontic robots allow painless tooth uprighting, rotating, and vertical repositioning, as well as rapid tissue repair. A new stainless-steel wire that uses nanotechnology is being studied that combines ultra-high strength with good deformability, corrosion resistance, and surface finish ${ }^{11}$.

\section{Nanoneedles}

Nanosized stainless-steel crystals incorporated into suture needles have been developed. Cell surgery may be possible in the near future with nanotweezers, which are now under development ${ }^{11}$.

\section{Nanocomposites}

Microfillers in composites and microcore materials have long been in use. Although the filler particle size cannot be reduced below $100 \mathrm{~nm}$, nanocomposite particles are minute enough to be synthesized at the molecular level. These nanoparticles improve the compressive strength of the material. Filler particles of submicron size, such as zirconium dioxide, are also necessary to improve polishability and esthetics. However, when particles of this size are used, the material may be more prone to brittleness and cracking or fracturing after curing.

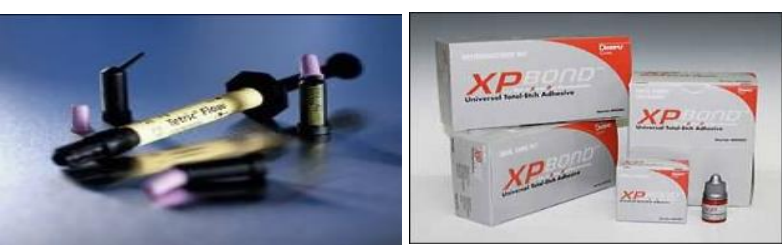

Figure 9: Nanocomposites

To address this issue, hybrid composites and composites containing a wider distribution of filler particles have come into use. Although these composites display a better balance of strength and esthetics, they are weak due to nanoparticle clumping or agglomeration. This problem can be overcome by incorporating a proprietary coating process during the particle manufacturing procedure, thereby eliminating weak spots and providing consistent strength throughout the entire "fill" of the core build-up. Additionally, the even distribution of nanoparticles results in a smoother, creamier consistency and improves flow characteristics. Once the material is cured to its hardened state, these properties contribute to the dentin-like cutability and polishability of the material (Kumar and Vijayalakshmi, 2006 ${ }^{13}$; Abhilash, 2010) ${ }^{15}$.

\section{Major tooth repair/nanotissue engineering}

Replacement of the whole tooth, including the cellular and mineral components, is referred to as complete dentition replacement. This therapy is possible through a combination of nanotechnology, genetic engineering, and tissue engineering. Complete dentition replacement was the basis for research by Chan et al., who recreated dental enamel, the hardest tissue in the human body, by using highly organized microarchitectural units of nanorods ${ }^{11}$.

14. Dental implants: structure, chemistry, and biocompatibility

The determining factors for successful osseointegration are surface contact area and surface topography. However, bone bonding and stability also play a role. Bone growth and increased predictability can be effectively expedited with implants by using nanotechnology. The addition of nanoscale deposits of hydroxyapatite and calcium phosphate creates a more complex implant surface for osteoblast formation (Albrektsson et al., 2008 ${ }^{16}$; Goene et al., 2007 ${ }^{17}$ ). Extensive research on the effects and subsequent optimization of microtopography and surface chemistry has produced ground-breaking strides in material engineering. These new implants are more acceptable, because they enhance the integration of nanocoatings resembling biological materials to the tissues ${ }^{11}$.

\section{Application of Carbon Nanotubes (CNT) in oral regenerative medicine ${ }^{6}$}

There is no doubt that dentistry is one of the branches of medicine most interested in the application of new knowledge acquired from the recent advances in tissue engineering and, particularly, in the field of nanotechnology. Important areas such as implantology, periodontics, and oral and maxillofacial surgery can benefit tremendously from the development of new nanomaterials. In the past few years, CNT combined with biopolymers have been novel potential biomaterials that may aid in the restoration of bone defects in dentistry. Currently, much information about the 


\section{International Journal of Science and Research (IJSR) \\ ISSN (Online): 2319-7064}

Index Copernicus Value (2013): 6.14 | Impact Factor (2014): 5.611

synthesis, characterization, and properties of CNT is available; however, more in vitro and in vivo studies are needed for a better comprehension of their effects on bone repair/regeneration. In 2010, Mendes et $\boldsymbol{a l}^{18}$. evaluated the effects of single-walled CNT (SWCNT) associated with sodium hyaluronate (HY-SWCNT) on bone repair/ regeneration of tooth sockets in rats. Treatment of sockets with this nanomaterial increased the formation of bone trabeculae approximately 3-fold and decreased the number of cell nuclei, thus indicating that the healing process was advanced in comparison with that in control sockets. Also, the expression of type I collagen was increased by $46 \%$ in treated sockets after 7 days of tooth extraction. This is an important finding, since, during the bone healing process, the collagen fibrils form osteoid, which allows for the deposition of crystals of carbonated hydroxyapatite responsible for mineralization of the bone matrix (Bouletreau et al., 2002) $^{19}$. Thus, an augmented expression of collagen type I in HY-SWCNT-treated sockets further suggests that the healing process is accelerated in these sockets (Mendes et al., 2010) ${ }^{18}$. No evidence of toxicity was observed in this study (Mendes et al., 2010). Similar methodology was used to investigate the effects of HYSWCNT on bone repair/regeneration of tooth sockets of rats under conditions in which bone repair/regeneration was hindered. It is well-known that diabetes alters bone metabolism by reducing both neoformation and resorption, prolonging the process of bone tissue repair/regeneration. Thus, the effects of HY-SWCNT were tested in rats with type I diabetes, induced by streptozotocin (Sá et al., 2013) ${ }^{20}$. It was found that, 14 days after tooth extraction, the bone repair/regeneration was approximately 3.3-fold higher in tooth sockets of diabetic rats treated with the nanomaterial. The treatment markedly increased the formation of bone trabeculae and reduced the number of cell nuclei, reaching values similar to those observed in non-diabetic rats. Therefore, the treatment with HY-SWCNT was able to restore the bone repair/regeneration process in tooth sockets of diabetic rats (Sá et al., 2013). In summary, both of the above-described studies (Mendes et al., 2010; Sá et al., 2013) verified that HY-SWCNT have great potential use for bone regenerative procedures in dentistry, in normal and adverse metabolic states. Corroborating these findings, Usui et al. (2008) ${ }^{21}$ conducted a series of experiments implanting MWCNT into different types of bone in mice. First, they implanted MWCNT subperiosteally into the skull to evaluate bone-tissue compatibility. At 1 and 4 wks postsurgery, there were no significant signs of incompatibility. These authors then implanted MWCNT into tibial defects to examine their influence on bone healing during $4 \mathrm{wks}$ and found a bone formation pattern similar to that of the control group, suggesting that the nanomaterial did not inhibit bone regeneration. More importantly, MWCNT particles promoted immediate HA crystallization on their surfaces, acting as a core for initial HA crystallization ${ }^{21}$.

\section{Challenges faced by nanodentistry ${ }^{3}$}

\section{Engineering challenges}

- Feasibility of mass production technique

- Precise positioning and assembly of molecular scale part

- Manipulating and coordinating activities of large numbers of independent microscale robots simultaneously.

\section{Biological challenges}

- Developing biofriendly nanomaterial Ensuring compatibility with all intricate of human body

\section{Social challenges \\ - Ethics \\ - Public acceptance \\ - Regulation and human safety}

Engineering challenges: There is a problem in the feasibility of mass production technique. Precise positioning and assembly of molecular scale part is a challenge for them. Manipulating and coordinating activities of large numbers of independent microscale robots simultaneously is a difficult task. Even though the field of nanorobotics is fundamentally different from that of the macro robots due to the differences in scale and material, there are many similarities in design and control techniques that eventually could be projected and applied. Due to the modern scientific capabilities, it has become possible to attempt the creation of nanorobotic devices and interface them with the macro world for control. There are countless such machines which exist in nature and there is an opportunity to build more of them by mimicking nature. Now a days these nanorobots play a vital role in the field of Bio Medicine \& dentistry, especially in the treatment of cancer. It also helps to remove the defected part in our DNA structure and some other treatments that have the greatest aid to save human lives.

Biological challenges: It is essential to develop bio friendly nanomaterial and ensure compatibility with all intricate of human body. In general, smaller particles are more bioactive and toxic. Their ability to interact with other living systems increases because they can easily cross the skin, lung, and in some cases the blood/brain barriers. Once inside the body, there may be further biochemical reactions like the creation of free radicals that damage cells. While the body has builtdefense for natural particles it encounters, the danger of nanotechnology is that it is introducing entirely new type of particles. Some experts believe that the body is likely to find toxic. Social challenges: Highest at risk are workers employed by manufacturers producing products that contain nanoparticles. The National Institute for Occupational Safety and Health (NIOSH) reports over two million Americans are exposed to high levels of nanoparticles. NIOSH publishes safety guidelines and other information for those employed in the nanoindustry ${ }^{3}$.

\section{Nano-Hazards ${ }^{5}$}

Since nanotechnology is a very recent discovery and is only just being put in to use, there are issues that need to be addressed. As long term effects of nanotechnology are unknown, therefore, potential hazards caused by the nanotechnology might not show for many years. Various factors govern the amount of free Nanoparticles in nature such as their physic-chemical properties, quantity, and time of exposure. Nanomaterials released in the environment can be further modified by, Temperature, $\mathrm{pH}$, different biological conditions, and presence of other pollutants. In this interaction, nanomaterials can alter atmosphere, soil and water and prove to be harmful to human health and the environment ${ }^{5}$. 


\section{International Journal of Science and Research (IJSR) \\ ISSN (Online): 2319-7064 \\ Index Copernicus Value (2013): 6.14 | Impact Factor (2014): 5.611}

It has been reported that nanomaterials can enter the human body through several ports. Accidental or involuntary contact during production or use is most likely to occur via the lungs and skin, from which a rapid translocation is possible to other vital organs through the bloodstream. Carbon black Nanoparticles have been implicated in interfering with cell signalling. It could also have unwanted effects on the DNA of cells, which could potentially cause genetic defects if this were to happen it would take a lot of time and research to put right. There is a need for developing systemic solutions, monitoring, and recording of the potential hazard as well as finding timely responses in order to achieve safety for human health and the environment ${ }^{5}$.

\section{Conclusion}

Nanodentistry has strong potential to revolutionize dentistry to diagnose and treat diseases. Dr. Gregory Fahy described nanorobots as "living organisms, naturally existing, fabulously complex systems of molecular nanotechnology", Nanotechnology will change dentistry, healthcare, and human life more profoundly than many developments of the past. As with all technologies, nanotechnology carries a significant potential for misuse and abuse on a scale and scope never seen before. However, they also have potential to bring about significant benefits, such as improved health, better use of natural resources, and reduced environmental pollution. Current work is focused on the recent developments, particularly of nanoparticles and nanotubes for periodontal management, the materials developed from such as the hollow nanospheres, core shell structures, nanocomposites, nanoporous materials, and nanomembranes will play a growing role in materials development for the dental industry ${ }^{22}$. Nanomedicine needs to overcome the challenges for its application, to improve the understanding of pathophysiologic basis of disease, bring more sophisticated diagnostic opportunities, and yield more effective therapies and preventive properties ${ }^{7}$. Molecular technology is destined to become the core technology underlying all of 21st century medicine and dentistry ${ }^{23}$.

\section{References}

[1] Freitas RA Jr. Nanodentistry. J Am Dent Assoc 2000;131(11):1559-65.

[2] West JL, Halas NJ. Applications of nanotechnology to biotechnology commentary. Curr Opin Biotechnol 2000;11:215-7.

[3] Bumb SS, Bhaskar DJ, Punia H. Nanorobots \& challenges; faced by nanodentistry. General. Sept 2013.

[4] Kanaparthy R, Kanaparthy A. The changing face of dentistry: nanotechnology. Int J Nanomed 2011;6;2799804.

[5] Bharath N, Gayathri G.V., D.S. Mehta. Nanorobotics in Dentistry- The Present Status And Future Perspective. Journal of Dental Practice and Research . 2013:1; (2); 41-47.

[6] Martins P.A, C.E. Alcântara, R.R. Resende, and A.J. Ferreira. Carbon Nanotubes: Directions and Perspectives in Oral Regenerative Medicine. J Dent Res 92(7):575-583, 2013.

[7] Freitas R.A. Nanodent. J. Am. Dent. Assoc. 2000;131(3):1559-1566.
[8] Lampton C. Nanotechnology promises to revolutionize the diagnosis and treatment of diseases. Genet. Eng. News. 1995;15:23-25.

[9] Song J.M., Kasili P.M., Griffin G.D., Vo-Dinh T. Detection of cytochrome $\mathrm{C}$ in a single cell using an optical nanobiosensor. Anal. Chem. 2004;76(9):25912594.

[10]Wong D.T. Salivary diagnostics powered by nanotechnologies, proteomics and genomics. J. Am. Dent. Assoc. 2006;137:313-321.

[11] Shetty NJ, Swati P. David K Nanorobots: Future in dentistry. The Saudi Dental Journal2013; 25: 49-52.

[12] Nagpal Archana, Kaur Jasjit, Sharma Shuchita, Bansal Aarti, Sachdev Priyanka. Nanotechnology - the era of molecular dentistry. Indian J. Dent. Sci. December 2011;3(5).

[13] Kumar S.R., Vijayalakshmi R. Nanotechnology in dentistry. Indian J. Dent. Res. 2006;17:62-69.

[14] Webster TJ, Ergun C, Doremus RH, Siegel RW, Bizios R. Enhanced osteoclast like cell functions on nanophase ceramics. Biomaterials 2001;22:1327-33.

[15] Abhilash M. Nanorobots. Int. J. Pharma Bio. Sci. 2010;1(1):1-10.

[16] Albrektsson T., Sennerby L., Wennerberg A. State of the art of oral implants. Periodontology. 2008;47:15-26.

[17] Goene R.J., Testori T., Trisi P. Influence of a nanometerscale surface enhancement on de novo bone formation on titanium implants: a histomorphometric study in human maxillae. Int. J. Periodontics Restor. Dent. 2007;27:211-219.

[18] Mendes RM, Silva GA, Caliari MV, Silva EE, Ladeira LO, Ferreira AJ (2010). Effects of single wall carbon nanotubes and its functionalization with sodium hyaluronate on bone repair. Life Sci 87:215-222.

[19] Bouletreau PJ, Warren SM, Longaker MT (2002). The molecular biology of distraction osteogenesis. $J$ Craniomaxillofac Surg 30:1-11.

[20] Sá M, Andrade V, Mendes R, Caliari M, Ladeira L, Silva E, et al. (2013). Carbon nanotubes functionalized with sodium hyaluronate restore bone repair in diabetic rat sockets. Oral Dis [Epub ahead of print 9/28/2012].

[21] Usui Y, Aoki K, Narita N, Murakami N, Nakamura I, Nakamura K, et al. (2008). Carbon nanotubes with high bone-tissue compatibility and bone-formation acceleration effects. Small 4:240-246.

[22] Patil M, Mehta DS, Guvva S. Future impact of nanotechnology on medicine and dentistry. J Indian Soc Periodontol. 2008;12(2):34-40.

[23] Kukreja BJ, Dodwad V, Singh T. Robotic dentistry- the future is at the horizon. Jounal of Pharmaceutical and biomedical sciences. 2012;16(1):1-4. 